\title{
Advanced Monitoring Systems for Smart Tooling in Aeronautical Industry 4.0
}

\author{
by P. Venegas*, G. Durana**, J. Zubia**, I. Sáez de Ocáriz* \\ * Aeronautical Technologies Centre, Juan de la Cierva 1, 01510 Miñano (Spain), pablo.venegas@ctaero.com \\ ** University of the Basque Country, Applied Photonics Group, Paseo Rafael Moreno Pitxitxi, 48013 Bilbao \\ (Spain), gaizka.durana@ehu.eus
}

\begin{abstract}
Historically, aeronautical engineering specifies demanding requirements in terms of precision, inspection and calibration during the assembly and construction of aircrafts. These requirements together with the present needs for more efficient and automated processes make it necessary to explore new concepts of intelligent tools capable of feeling and acting, integrated within the Industry 4.0. Based on these assumptions, this study aims for the development of monitoring strategies based on infrared radiation and high precision fibre optical sensors to monitor critical parameters of the structures to be used as input to control models of adaptronic systems.
\end{abstract}

\section{Introduction}

The air transport of passengers and cargo is one of the main engines of the world economy and a strategic sector at the country level. Despite the recent crisis, the global fleet of commercial aircraft and the number of purchase orders have increased in that period and independent estimates indicate for the 2016-2035 period that 42,800 new commercial transport planes, turbojets and turboprops will be delivered, both passengers and cargo, with an estimated value of about 3,000 billion dollars [1].

An important strategic objective proposed by the European aeronautical sector is the improvement of competitiveness. To achieve this goal, aerostructures companies are working hard on new designs of complex aerostructural components and on process optimization and automation. Among all the processes, the assembly operations of an aircraft account for more than $40 \%$ of the work in its manufacture. These operations have a considerable impact on the final quality of the aircraft and its cost and manufacturing cycle.

The tooling currently used for manufacturing and assembly processes, machining and testing of large aerostructural components must meet critical requirements (positioning, rigidities, etc.), being made ad-hoc for each component, and not adaptable to a family of products or variations in the production rate. In most cases, similar designs have been used for 50 years. They are rigid and heavy with a high energy cost in operation. They do not adapt to the environment, being passive, spacious and costly elements.

Current conceptions of automation demanded by the aeronautical sector imply flexibility for multiple products, multiple processes and multiple programs, so this type of traditional solutions is not valid. Ongoing R\&D projects propose a conceptual change in the tools currently used, with the main objective of developing a new concept of intelligent, modular and flexible tooling for assembling, machining and testing large complex aerostructures, as a driving element for the automation of the Aeronautical industry 4.0. These intelligent tooling integrates monitoring and actuation systems, enabling sensing and acting in real time during the assembly, machining and testing processes of such components to adapt to specific necessities. This study focuses on the development of monitoring technologies to provide sensory faculties to new tooling concepts. These technologies are Infrared Thermographic [2,3] and Fibre Optic Sensors [4,5].

\section{Monitoring requirements}

The main events that occur in structural elements of aeronautical tools for assembly and testing have been identified with the aim of developing monitoring strategies, based on IR radiation measurements and fibre optic sensors, The measures representative of the thermal and tensional state of the structure will serve as input to the control models of the adaptronic system to act accordingly and prevent damage to the structures and the mounted components. The criteria applied for selecting these events were both the frequency of occurrence and the impact on the integrity of the structure. The objective is to simulate them at laboratory level to identify the thermal and stress effects that produce, in their different degrees of severity, in order to classify and correlate to the final outcome for later management through the control system. By analysing the main characteristics of these events, it will be possible to classify the types of failures that have occurred, obtaining the necessary information to undertake the classification process with guarantees.

The identified incidents are the appearance and propagation of defects, local dilatations, accidental contacts and overloading, among others. These events are directly related to common failures that originate in these structures such as appearance and propagation of cracks in metal materials, break of joints, appearance and propagation of delaminations in composite materials, break of polymer matrix and debonding. 

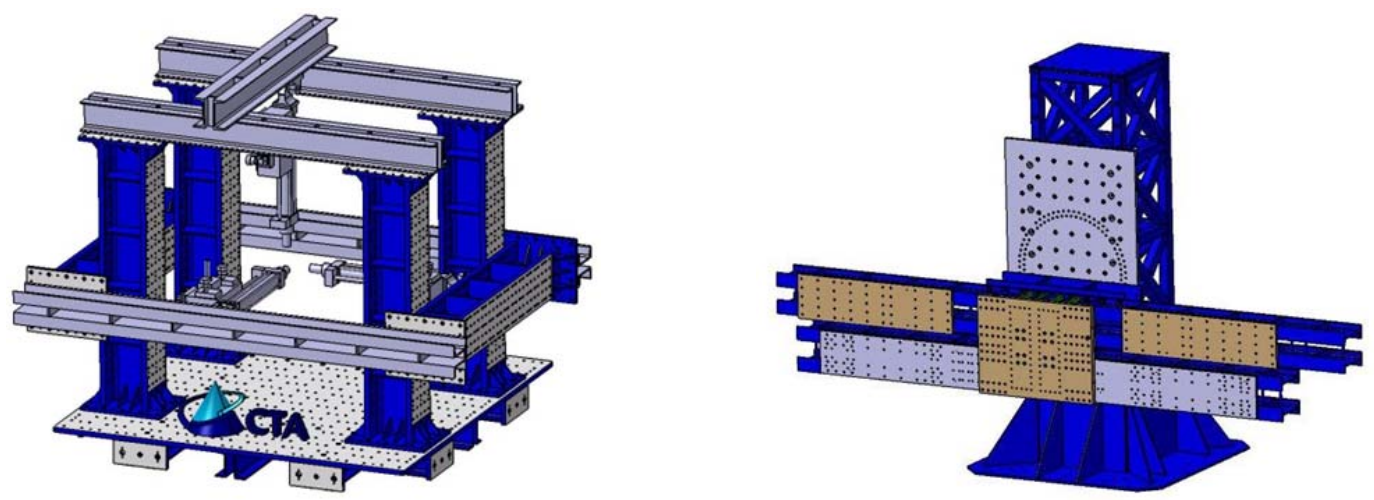

Fig. 1. Examples of conventional tooling made of rigid steel profiles assembled by screws.

. The detailed analysis of the behavior of these events will allow classifying the types of failures that occurred, obtaining the necessary information to automate the classification process. From a thermal point of view, the characteristics of greatest interest to be analyzed in the laboratory are the energy level reached, the duration of the event, the affected area and the line of propagation.

The particular characteristics of infrared thermography (IRT) to monitor remote processes and without contact make this technology a tool of great potential for the development of intelligent tooling. Infrared thermography used as a monitoring system for components subjected to structural load states offers great advantages over conventional monitoring systems and whose development will serve to increase the level of quality of the corresponding processes, reducing time and associated costs.

Fiber Bragg Grating (FBG) sensors are contact sensor that have demonstrated high performance in characterizing the thermal and stress state of materials. A fiber Bragg grating (FBG) is a type of distributed Bragg reflector constructed in a short segment of optical fiber that reflects particular wavelengths of light and transmits all others. This is achieved by creating a periodic variation in the refractive index of the fiber core, which generates a wavelength-specific dielectric mirror. A fiber Bragg grating can therefore be used as an inline optical filter to block certain wavelengths, or as a wavelength-specific reflector.

\section{Manufactured samples and Monitoring technologies}

A set of specimens have been designed and manufactured in material commonly used in the structures under study, metal and/or composite, considering advanced tooling concepts designs $[6,7]$. There is a great variety of materials with which the aeronautical tools and components are manufactured. These components have different levels of complexity and may consist of simple metal plates or complex assemblies of numerous elements joined together. Within this wide range of possible material structures, the study has focused on those that are of greater interest because of their high frequency of use or because of their high manufacturing and/or repair costs. The materials selected for the characterization of thermal events have been steels type S355, 42CrMo4, aluminum alloy type 7075 T651 and epoxy composite materials/carbon fiber. Drills, impacts and cracks have been made in the manufactured specimens to simulate defects and the typical geometry of the elements they represent. The tests have been carried out in a TractionCompression machine available at CTA, applying to each specimen different tensional and thermal states produced by the application of static charges, dynamic loads, small impacts and localized heating.

In order to develop non-contact monitoring strategies based on IR radiation measurements, the main types of events that occur in structural elements of aeronautical assembly and test tooling have been simulated experimentally at laboratory level, and the thermal and stress effects have been analyzed using thermographic and FBG sensors, enabling to establish cause-effect relationships and define strategies to be carried out with advanced control systems. Infrared images were acquired using a FLIR SC5500 camera. This camera is equipped with a cooled Indium antimonide detector that operates in the 2.5-5.1 mm waveband. The FLIR SC5500 produces thermal images of 320x256 pixels with 12 bits per pixel and a thermal sensitivity of $20 \mathrm{mK}$. Although the camera has a maximum frame rate of $383 \mathrm{~Hz}$ at full-frame, the experiments were recorded at $50 \mathrm{~Hz}$ to reduce size of acquired videos. The integration time was set to $4000 \mu s$ to capture the low IR radiation levels during the tests. The FBG sensors used in the study were Micron Optics os3110 optical strain gauges with wavelength of approximately $1550 \mathrm{~nm}$ and strain range of $\pm 2500 \mu \varepsilon$. To compensate the stress due to thermal dilatation one temperature FBG was used, Micron Optics os4100 optical strain gauges with wavelength of approximately $1550 \mathrm{~nm}$ and temperature range between -40 to $+120^{\circ} \mathrm{C}$.

Traction-Compression machine used to produce the thermal and stress conditions to the samples incorporates a hydraulic actuator capable of applying up to $500 \mathrm{KN}$ load at static and dynamic regimes. 


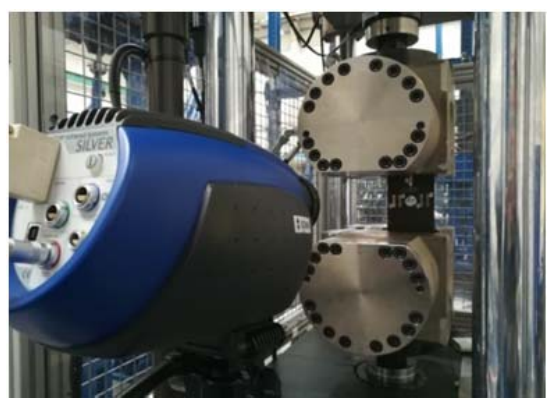

a)

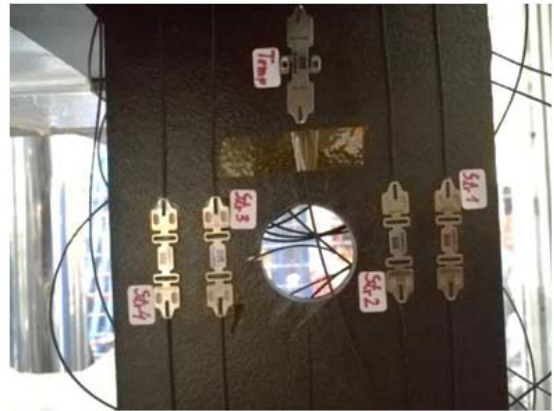

b)

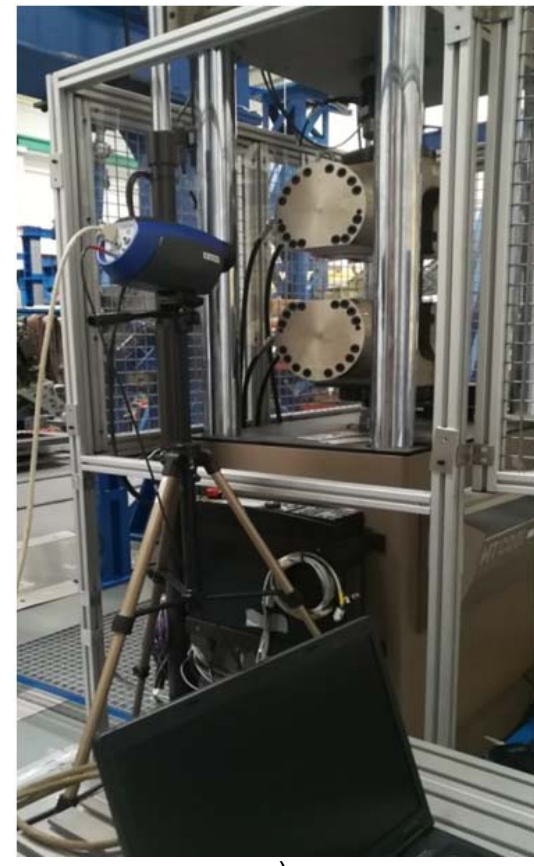

c)

Fig. 2. Images of the preliminary test campaign. (a,c) Detail and general IRT monitoring setup, (b) positions of FBG sensors in a CFRP sample.

\section{Tests campaign and Preliminary results}

A set of test samples with the previously defined materials and configurations have been manufactured and used in the thermal event characterization tasks. A preliminary tests campaign has been carried out under laboratory conditions for the development of non-contact monitoring strategies

IRT and FBG sensors have been used to measure the instantaneous temperature magnitudes, deformations/stresses, positions and growth of simulated defects. The preliminary results of this testing campaign have provided relevant information for the characterization of events and their use in the advanced control of smart tooling.

\subsection{Localized heating areas}

This type of tests simulates the temperature increase in the tooling elements as a result of heat transmission from surroundings due to works being conducted such as assembly or machining of the mounted component. This event may be critical if the dilatation produced by the overheating makes the tooling dimensions change enough to go beyond the required dimensional tolerances. The objective of these tests is to define operative protocols capable of quickly detecting these overheating and produce information to input a control system that would counteract with a controlled readjustment of the moving parts of the tooling.

The parameters used in this type of tests try to simulate the actual conditions that exist in a real tooling. Therefore both temperature and stress levels were considered taking three different load levels and three different heat application points. The load levels were 10, 20 and $30 \mathrm{KN}$, that produced stress levels between 10 and $50 \mathrm{MPa}$ depending on the section of the tested sample, and the heat was produced by a hot air gun with the output air temperature set to $100^{\circ} \mathrm{C}$ applied during 2 seconds in three different points of the sample located at different distances to the temperature FBG sensor

The results obtained show that IRT sensor is capable of monitoring the thermal evolution of the material with high precision and spatial resolution so any superficial overheated point may be rapidly detected. On the other hand the temperature FBG is also able to accurately measure temperature values but the measuring area is limited to a restricted zone occupied by the fiber. An important incompatibility between both technologies was identified in the tests, which consists in that the FBG sensor plate hides partially the surface under monitoring so the IRT sensor fails in those areas (figure 3).

The preload applied to the samples produced no effect in the temperature measurements as long as the sample is at stress equilibrium. The load application produces thermal effects different from those ones due to the heat injection so they can be accurately measured when applied separately. Therefore, the heat was applied 5 minutes after the stress level was set to the sample in order to reach thermoelastic equilibrium. 

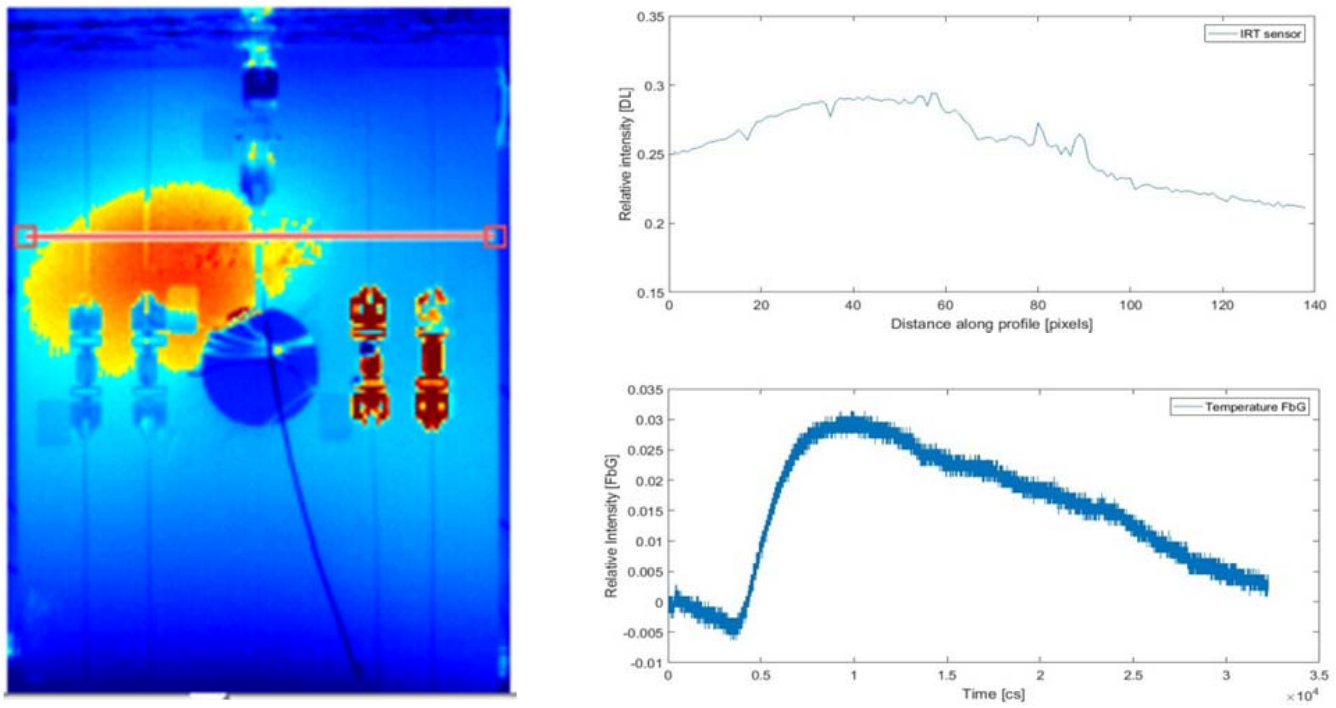

Fig. 3. Results obtained by IRT (left and top right) and FBG (bottom right) sensors in the tests for localized heating areas.

\subsection{Low energy vibrations}

This type of tests simulates the vibrations that affect the tooling elements, produced by tasks conducted to the mounted components such as drilling, milling or edge finishing. This events may be critical since the vibrations applied together with static loads tends to loosen joints and create defects, such as cracks, delaminations or debondings, as well as to propagate the existing ones. The objective in these tests is to define procedures for quick detection of defects and growth monitoring in order to specify alerts and emergency stops to be triggered by the control system during tooling operations.

Since the stress is greater in concentration points such as holes and areas of curved geometry, these points are more prone to experience damage occurrence and tightening failures. Drills and notches have been made in the tested specimens in order to monitor these stress concentration zones and damage growth. The control of the stress values was carried out through the relationship between stress and temperature variation by the thermoelastic effect for the IRT sensors $[8,9]$ and through the relationship between stress and strain by the elasticity equation for the FBG sensors. The damage growth was quantified by thermal image correlation techniques.

The load levels applied were 10, 20 and $30 \mathrm{KN}$ with frequencies of 2 and $6 \mathrm{~Hz}$. FBG sensors used the material mechanical parameters to relate the measured strains values with the current stress levels and is calculated by the elasticity equation (1), where $\varepsilon$ is the strain tensor, $\sigma$ is the stress tensor, $C$ is the elasticity matrix and $\alpha$ the thermal expansion coefficients matrix. Even though the stress levels were low the FBG sensors produced high quality signals that enabled to obtain accurate measures.

$$
\{\varepsilon\}=[C]\{\sigma\}+\Delta T\{\alpha\}
$$

IRT sensor used the mechanical and thermal parameters of the material under inspection to relate the measured thermal values to the current stress levels. This relation is established by the thermoelastic equation (2), valid for adiabatic processes, where $\varepsilon_{i j}$ are the components of the strain tensor, $\sigma_{i j}$ the components of the stress tensor, $C_{\sigma}$ is the specific heat at constant pressure and $T$ the absolute temperature.

$$
\rho C_{\sigma} \frac{d T}{T}=-\frac{\partial \varepsilon_{i j}}{\partial T} d \sigma_{i j}
$$

The quality of the thermal signal measured by the IRT sensor was reduced due to the low stress levels induced in the samples by the introduced vibrations. The accuracy provided by the direct application of equation (2) was very limited. Therefore a Lockin process was used to estimate the extreme values. A computational algorithm was generated and employed to measure in real time the frequency of the applied vibrations so the amplitude value of the measured thermal signal was determined and the maximum stress value calculated. 

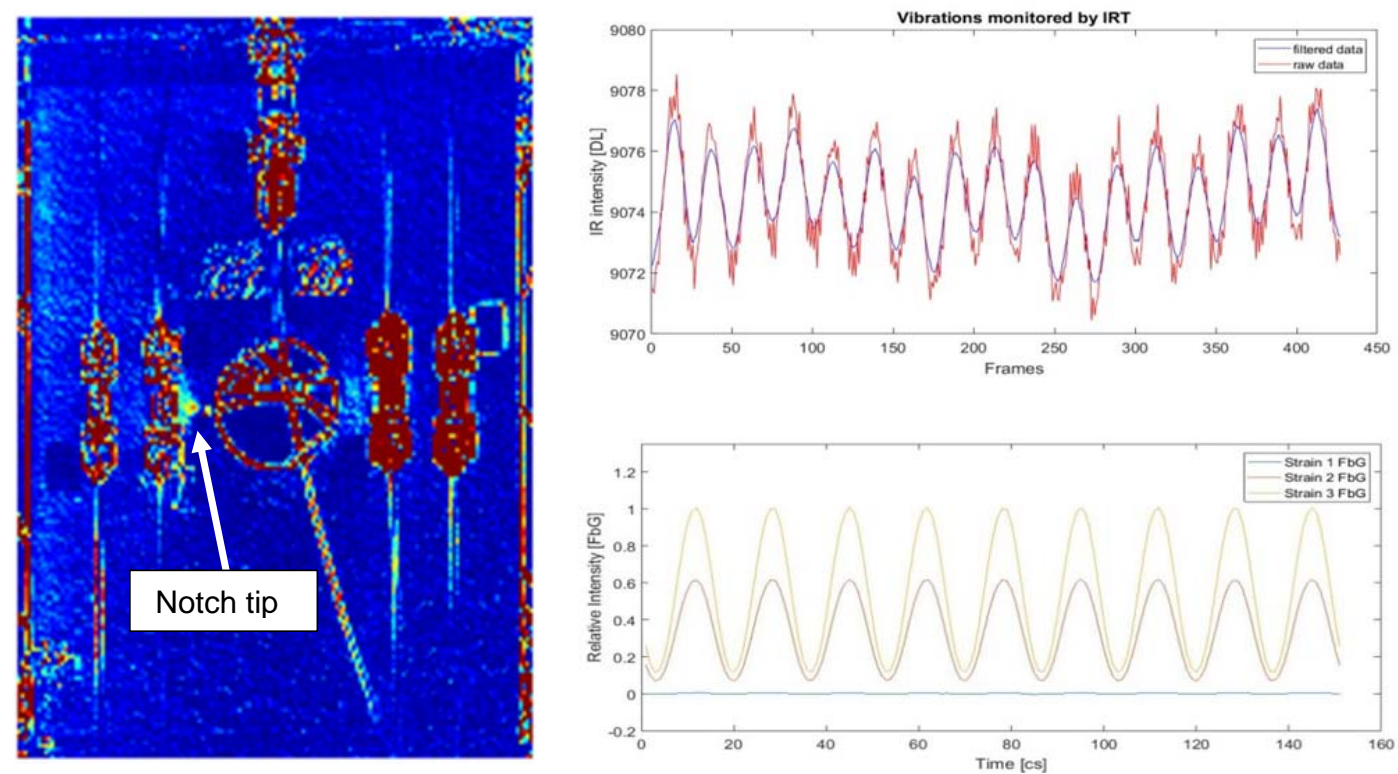

Fig. 4. Results obtained by IRT (left and top right) and FBG (bottom right) sensors in the low energy vibration tests.

The results obtained in this test campaign show that IRT sensor is capable of detecting thermal signal produced by the vibrations but the high noise levels hide the thermal data disabling to produce correct stress values. A preliminary filtering stage followed by a Lockin calculation procedure demonstrated to be a suitable protocol to recover the hidden information from data noise and produce improved qualitative stress values. FBG sensors demonstrated to be very robust and stable so they provide accurate measures of stress values under all the analyzed conditions.

The limitation of the measuring area in FBG sensors restricted to the zone occupied by the fiber was highlighted during the tests. It would be necessary to bond numerous FBG sensors to produce a stress map. On the other hand, IRT sensor finds limitations in accuracy but provides high spatial resolution so a qualitative stress map may be produced. Additionally, the growth of the damage may be visualized during the process (figure 4). FBG sensor plates hide partially the surface under monitoring so those areas are blind for IRT sensor and fails in following the damage growth.

\subsection{Static load applications}

Several events in the tooling may be simulated by this type of tests. Static load application simulates the adjustment of the tooling elements to the mounted component at the beginning of the assembly tasks as well as subsequent punctual forces applied to the mounted components due to activities such as drilling or assembly with other parts of the whole component.

These events may be critical since static loads tend to deform the tooling elements so the relative positions of the mounted components are consequently affected and dimensional tolerances may be exceeded. Wrong dimensional tolerances would produce the component to be discarded. Static loads also tend to loosen joints and create defects, such as cracks, delaminations or debondings, and even may produce sudden defect propagation till catastrophic failure. Moreover static loads overstress the tooling elements producing high deformations and in extreme situations plasticizing.

The objective in these tests is to detect static force increases due to external factors and measure the stress levels reached as a result of the applied loads. The stress state monitoring enables to define procedures for reacting appropriately to compensate them by actuation of the tooling elements so the assembled component is not affected and dimensional tolerances met. Furthermore, the quantification of the local stress state will enable to specify alerts and emergency stops to be triggered by the control system during tooling operations.

The load levels applied in this test campaign were $5,10,15,20$ and $25 \mathrm{KN}$, which produced stress levels between 5 and $45 \mathrm{MPa}$ depending on the section of the tested sample. The analysis methodology followed for IRT monitoring was to detect significant changes in the thermal state of the material by the IR sensor and estimate the corresponding stress increase by the equation (2) restricted to the interval of load change. The validity of this equation is conditioned to the compliance of the assumption that adiabatic conditions prevails during the load application. Since the duration of load applications is less than $0,2 \mathrm{~s}$, according to the T-CM control system, this condition may be accepted. 

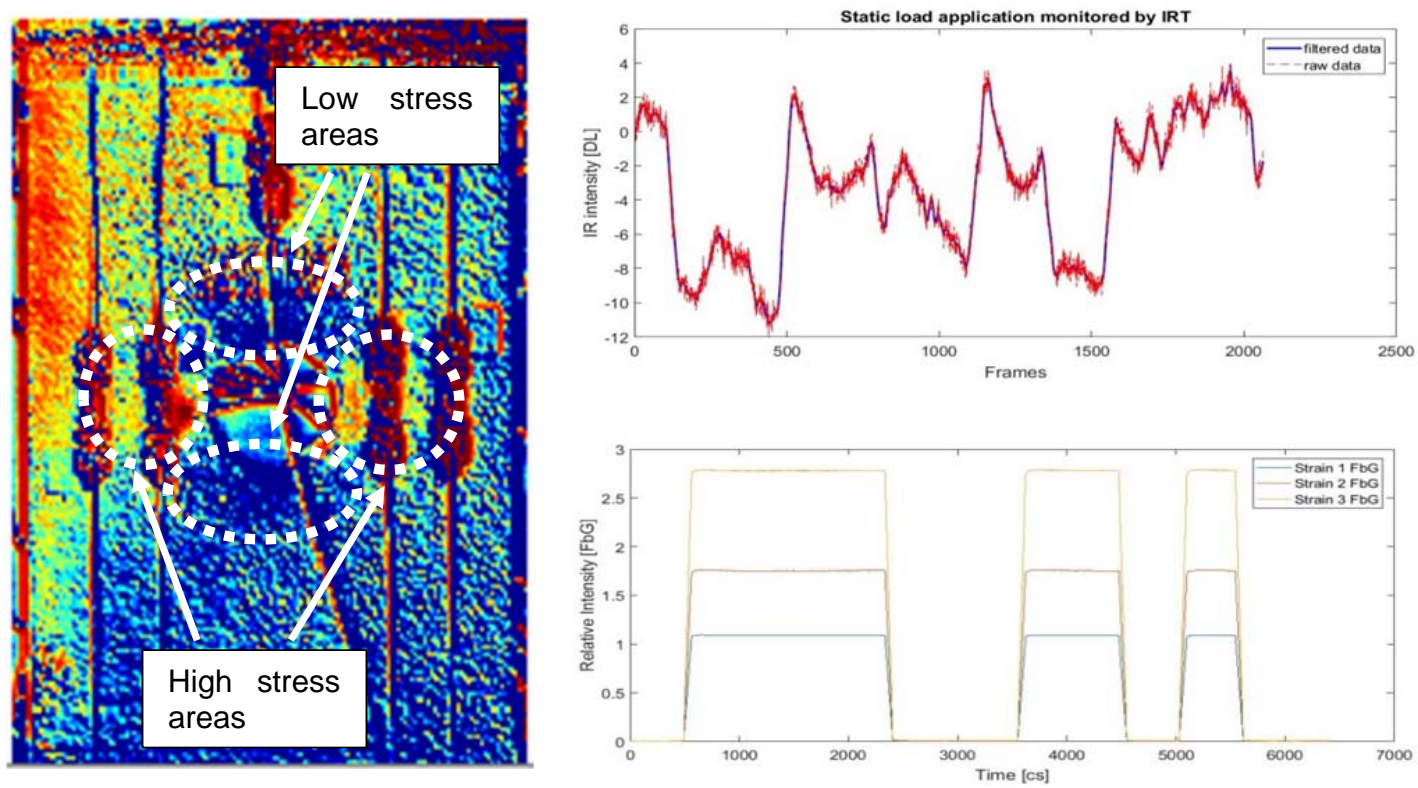

Fig. 5. Results obtained by IRT (left and top right) and FBG (bottom right) sensors in the static load tests.

The results obtained in this test campaign show that IRT sensor detects changes in thermal signal produced by load applications but contains high noise levels so accurate stress values are impractical. A preliminary filtering stage consisting in Gaussian smoothing by spatial convolution was applied to determine the changes in thermal state corresponding to the application of static loads. This procedure proved to be a suitable operation to recover the hidden information from data noise and improve qualitative stress values. The thermographic images show correct qualitative identification of stress distribution, detecting areas of high stress levels (reddish areas in figure 5) and stress-free areas (dark bluish areas in figure 5) around the stress concentrators. The intentional defects introduced in the samples were also accurately monitored during the test indicating the high concentration factor they produce in the stress state. FBG sensors produced robust and stable values so they provide accurate measure of stress values under all the analyzed conditions.

The values measured by the sensors during the test campaign were compared to results obtained by FEM analysis in the case of stress measures and to values produced by a thermocouple in the case of temperature increase. A summary of the maximum errors produced by both sensors in the different type of measures is shown in table 1.

Table 1. Summary of the maximum errors produced by the sensors in the measures during the tests.

\begin{tabular}{|c|c|c|c|}
\hline \multirow{2}{*}{} & \multicolumn{3}{|c|}{ MAX ERROR [\%] } \\
\cline { 2 - 4 } & $\begin{array}{c}\text { TEMPERATURE } \\
\text { (HEATING) }\end{array}$ & $\begin{array}{c}\text { SRESS } \\
\text { (STATIC LOAD) }\end{array}$ & $\begin{array}{c}\text { STRESS } \\
\text { (VIBRATIONS) }\end{array}$ \\
\hline IRT SENSOR & 1,5 & 9,4 & 7,2 \\
\hline FBG SENSORS & 4,8 & 1,5 & 1,6 \\
\hline
\end{tabular}

\section{Conclusions and future work}

Aeronautical industry requires demanding precisions, inspections and calibrations during the assembly and construction of aircrafts. These requirements together with the present needs for more efficient and automated processes make it necessary to explore new concepts of intelligent tools capable of feeling and acting, integrated within the Industry 4.0. The tooling currently used for manufacturing and assembly processes of aero-structural components are manufactured ad-hoc for each component, and cannot be adapted to a family of products or variations in the production rate. They are rigid and heavy with a high energy cost in operation.

Current research lines propose a conceptual change with the main objective of developing a new concept of intelligent, modular and flexible tooling for assembling, machining and testing large complex aerostructures. These intelligent tooling integrates monitoring and actuation systems, enabling sensing and acting in real time during the assembly, machining and testing processes to adapt to specific necessities. This study focuses on the development of monitoring technologies based on infrared radiation and high precision fibre optical sensors to provide sensory faculties to new tooling concepts. 
A preliminary test campaign has been conducted under controlled conditions simulating the main events occurring in activities carried out in aeronautical tooling, such as localized warming, low energy vibrations and static load applications. The results obtained showed the limitations and strengths of the mentioned monitoring technologies and proved the high compatibility between them. IRT sensor was capable of monitoring the thermal evolution in localized warming situations with high precision and spatial resolution while FBG sensors, although provided accurate temperature values, produced a limited measuring area restricted to the zone occupied by the fiber. IRT sensor was capable of detecting thermal signal produced by the vibrations and static loads but the high noise levels corrupted the thermal data, disabling to produce accurate stress values. A preliminary filtering stage, followed by a Lockin calculation procedure in the case of vibration analysis, produced data noise reduction and improved the quantitative stress values. FBG sensors demonstrated to be very robust and stable in all the analyzed situations providing accurate measures of stress and temperature values.

Next work will consist in the integration of both technologies into an advanced control system to make a tooling demonstrator sensitive and enable to produce actuation to counteract the input values according to specific references. The compatibility of both technologies has been validated so FBG sensors will provide accuracy in the measures and IRT will provide spatial resolution in a combined monitoring system.

The authors would like to acknowledge the financial support provided by the Department for Economic Development and Infrastructures of the Government of the Basque Country in the project AEROTRESNAK (Exp. Number: KK-2017/00033).

\section{REFERENCES}

[1] Flight Global-Ascend Fleet Forecast 2016-2035.

[2] Maldague, X. P. (2012). Nondestructive evaluation of materials by infrared thermography. Springer Science \& Business Media.

[3] Usamentiaga R, Venegas P, Guerediaga J, Vega L, Molleda J, Bulnes F. G. Infrared thermography for temperature measurement and non-destructive testing. Sensors. 2014;14(7):12305-12348.

[4] Zhai, T., et al., Piezoresistive and compression resistance relaxation behavior of water blown carbon nanotube/polyurethane composite foam. Composites Part A: Applied Science and Manufacturing. 2015;72:108114.

[5] Lu, N., et al., Highly Sensitive Skin-Mountable Strain Gauges Based Entirely on Elastomers. Advanced Functional Materials. 2012;22(19):4044-4050.

[6] $\mathrm{Bi}$ Z, Zhang W. Flexible fixture design and automation: review, issues and future directions. Int. J. Prod. Res. 2001;39(13):2867-2894.

[7] Boyle I, Rong Y, Brown D. A review and analysis of current computer-aided fixture design approaches. Robot. Comput. Integr. Manuf. 2011;27(1):1-12.

[8] Palumbo D, Galietti U. Characterisation of steel welded joints by infrared thermographic methods. Quantitative InfraRed Thermography Journal, 2014;11(1):29-42.

[9] Emery, T. R., Dulieu-Barton, J. M., Earl, J. S., \& Cunningham, P. R. (2008). A generalised approach to the calibration of orthotropic materials for thermoelastic stress analysis. Composites Science and Technology, 68(34), 743-752. 